\title{
Modeling the Human Aorta for MR-Driven Real-Time Virtual Endoscopy
}

\author{
Klaus J. Kirchberg ${ }^{1,2}$, Andreas Wimmer ${ }^{2}$, and Christine H. Lorenz ${ }^{1}$ \\ ${ }^{1}$ Siemens Corporate Research Inc., Princeton, NJ, USA \\ \{Klaus.Kirchberg, Christine.Lorenz\}@siemens.com \\ ${ }^{2}$ Chair for Pattern Recognition, University of Erlangen-Nuremberg, Germany \\ Andreas.Wimmer@informatik.uni-erlangen.de
}

\begin{abstract}
As interventional magnetic resonance imaging (iMRI) is getting closer to clinical practice, new means of visualization and navigation are required. We present an approach to create a virtual endoscopic view inside the human aorta in real-time. In our approach, defined crosssectional slices are acquired and segmented in a highly optimized fashion. A geometric shape model is fit to the segmentation points and continuously updated during the intervention. The physician can then view and navigate inside the structure to plan the intervention and get immediate feedback about the procedure. As a component of this system, this work focuses on the segmentation of the cross-sectional images and the fitting of the shape model. We present a real-time 2D segmentation implementation for this application domain and a model fitting scheme for a generalized cylinder (GC) model. For the latter we employ a new scheme for choosing the local reference frame.
\end{abstract}

\section{Introduction}

Magnetic resonance imaging (MRI) has been receiving growing attention as guidance modality for minimally invasive procedures. Unlike X-ray fluoroscopy or computed tomography (CT), interventional MRI does not expose patient or physician to potentially harmful ionizing radiation. Also MRI shows detailed soft tissue contrast.

Commercially available MR scanners and their user interfaces are mainly tailored to diagnostic tasks. Visualization of scanned image slices and volumes is optimized for visual inspection on a high-definition display. Interventional MRI on the other hand requires real-time presentation of the acquired image data in the most meaningful way for the physician. Different approaches to MR image visualization for interventional procedures have been proposed. Current techniques include 2D image display of single or multiple slices in real-time 112], 2D projections of magnetic resonance angiography (MRA) data 3] and 3D volume rendering [4].

As off-line diagnosis tool, virtual endoscopy has been investigated over the last 10 years and found its way into clinical practice, e.g. for the detection of polyps in the colon. In [5], the authors propose real-time virtual endoscopy as a new navigation tool for MR-guided interventions. In contrast to diagnostic virtual endoscopy, 


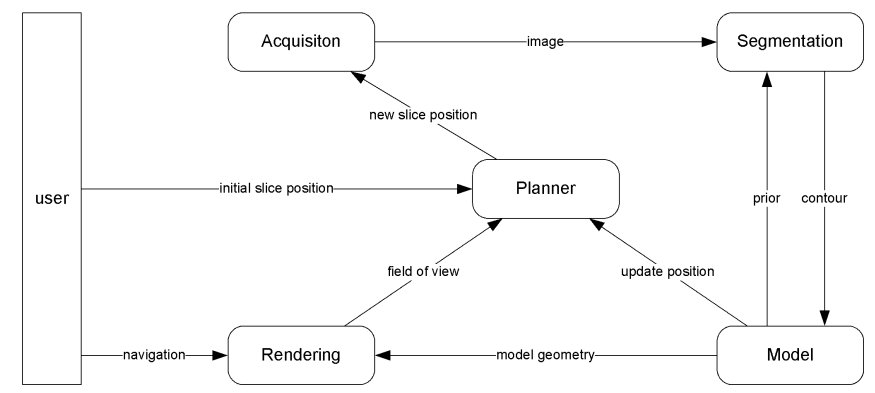

Fig. 1. Data flow in the real-time virtual endoscopy system

the $3 \mathrm{D}$ view reflects changes of the anatomy in real-time. The view is continuously updated using on-line MR acquisition, segmentation, and modeling.

In this work we focus on techniques for MR-guided interventions in the human aorta. Coarctation (narrowing) and aneurysms (enlargements) are examples of aortic defects that can be treated by minimally invasive catheter based procedures under X-ray or MRI guidance. During the treatment, a stent graft is inserted and deployed at the appropriate position. We hypothesize that 3D monitoring methods can help improve the accuracy of stent placement as well as reduce the chance of accidental tissue rupture, and improve early detection of complications.

For real-time interaction with the MR scanner we used a communication framework as described in [5]. It provides means of changing slice position and orientation during scanning as well as sending the resulting image data to the processing system.

The planned workflow is as follows. Image slices are acquired perpendicular to the aorta's main axis. The scanned images are first subject to segmentation. Here, points on the aortic wall are extracted and their 3D position is determined by applying the (known) transformation of the image slice. After segmentation, the $3 \mathrm{D}$ coordinates of the contour points are added to the cloud of data points. An optimization is continuously fitting and updating the aorta model to best match the point cloud. The coherence information of contour points within one slice is not necessary in our approach, thus leading to a more general system that can easily be extended to other organs. Using the current model shape knowledge, new image slices are requested from the scanner. The layout of the system is depicted in figure 1 .

Therefore, as a component of such a real time interventional system, we present methods for (1) optimized segmentation of the aorta and (2) a modified generalized cylinder (GC) model, both suitable for real time application. In particular, this work describes a GC model [6] for the human aorta that is usable in the real-time MRI scenario. A novel reference frame scheme is introduced to solve the problem of singularities in the Frenet-Serret frame. The presented approach is kept general, making it easily adaptable to other organs. 
We also present a highly optimized segmentation using Geodesic Active Contours suitable for real-time MRI.

\section{Method}

\subsection{Segmentation of the Aortic Cross-section}

Oriented roughly perpendicular to the aorta's main axis, the image slices of the healthy aorta exhibit a more or less circular cross-section. In case of a pathology, e.g. an aneurysm, the contour can diverge even to a concave shape. To handle this variability, segmentation is performed using a level set based approach.

The interventional scenario imposes severe constraints on acquisition and processing time. Feedback of the intervention should be less than a second. To achieve reasonable update detail the system should be able to acquire and process at least 5 frames per second with an in-plane resolution of about $2 \mathrm{~mm}$.

Description of the segmentation method. We segment the aortic crosssection using Geodesic Active Contours [7]. An initial curve $C^{0}$ (see below) is placed in the image and deformed by a curvature dependent speed $\kappa$ and a constant speed $\nu$ in curve normal direction $\mathbf{n}$ according to equation (1). $g$ is inversely related to the edge strength which is calculated from the input intensities I. It acts as a stopping function and slows down or stops the curve evolution at object boundaries. The second part of the right hand side in equation (11) constitutes an edge attraction speed in normal direction which increases the robustness for partially weak boundaries or boundaries with small gaps [7], a highly desirable property for images with reduced quality due to rapid acquisition times.

$$
\partial_{t} C=g(\kappa+\nu) \mathbf{n}-(\nabla g \cdot \mathbf{n}) \mathbf{n}
$$

We use the Perona-Malik diffusivity [8] given by equation (2) as stopping function $g . \lambda$ is a contrast parameter which controls the decrease of the stopping function with increasing edge strength. The gradient is approximated using the Sobel Operator.

$$
g(\mathbf{x})=\frac{1}{1+\left(\frac{|\nabla I(\mathbf{x})|}{\lambda}\right)^{2}} .
$$

The curve evolution defined in equation (1) is tracked by the level set framework according to equation (3) [7. The curve is embedded as the zero level set of the $2 \mathrm{D}$ level set function $\phi$. In contrast to an explicit parametric representation, this implicit representation does not require any (re-)parameterizations during the evolution.

$$
\partial_{t} \phi=|\nabla \phi|\left(\operatorname{div}\left(g \frac{\nabla \phi}{|\nabla \phi|}\right)+\nu g\right) .
$$

We use a semi-implicit numerical implementation of the level set PDE. This permits large time steps and thus only few updates of the level set function are required until the object boundaries are reached by the deforming curve. 


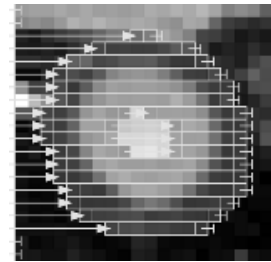

(a)

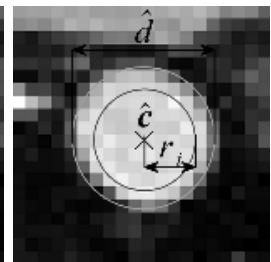

(b)

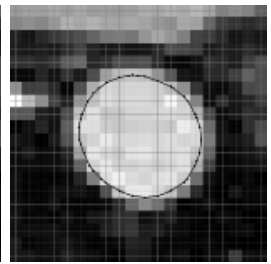

(c)

Fig. 2. (a) Linked list structure to maintain the narrow band rows (b) Placement of the initial curve $C^{0}$ (c) Extraction of the final curve. See text for details.

A semi-implicit formulation usually comes with the burden of a high computational effort per update. In [9, a discretization of equation (3) is proposed which allows the application of the Additive Operator Splitting technique [10]. Thereby the computational effort is drastically reduced, and an overall speedup of an order of magnitude compared to an explicit formulation of the level set PDE is reported.

The Narrow Band approach [1] is used to further increase the efficiency of the segmentation algorithm. The computational domain is restricted to grid points in a neighborhood around the zero level set, thus reducing the complexity from $O\left(N^{2}\right)$ to $O(N)$ for a $N \times N$ grid. We use the linked list structure proposed in 12 to maintain which grid points are part of the narrow band. Figure 2 (a) shows the structure describing the narrow band rows. A modified Chamfer distance transformation [13] is used to re-initialize the narrow band once the zero level set comes close to its boundary.

Placement of the initial curve. The initial curve $C^{0}$ is placed within the cross-section and expanded in order to recover the boundary. Given estimates $\hat{\mathbf{c}}$ and $\hat{d}$ on the center and approximate diameter of the cross-section from our model of the aorta, the initial curve is set to a circle around $\hat{\mathbf{c}}$, as illustrated in figure 2 (b). The radius $r_{i}$ is set to a smaller value than half the diameter $\hat{d}$ in order to ensure that the initial curve is within the cross-section also for inaccurate estimates.

Extraction of the final curve. The level set function takes a steady state when the deforming curve has reached the object boundaries. The final curve is then extracted in sub-grid accuracy using the Marching Squares algorithm (see figure 2 (c)).

\subsection{Aorta Model}

Geometric shape models and their application in medical image analysis have been studied extensively. A survey of methods can be found in [14].

A common deformable model for cylindrical structures is the generalized cylinder model [6]. A generalized cylinder consists of a 3D space curve, called spine or centerline, and a variable $2 \mathrm{D}$ cross-section function. The modeled surface is then defined by sweeping the cross-section function along the spine using some sweep 
rules. However, this formulation has several weaknesses, e.g. the occurrence of discontinuities at certain points. To overcome those problems, several extensions have been proposed, e.g. [15|16].

We employ a circular cross-section function. This scheme models the healthy aorta as well as aortic abnormalities such as coarctation or aneurysm reasonably well while providing numerical stability for the model optimization process detailed below. In order to fit the model to the segmented surface points we define a cost functional that measures the quality of the fit. We then derive the Euler-Lagrange equation and perform the optimization by gradient descent.

Equation 4 depicts the cost functional, where $\mathbf{c}:[0,1] \mapsto \mathbb{R}^{3}$ defines the centerline curve and $r:[0,1] \mapsto \mathbb{R}$ the radius function of the cross-section parametrized over the same interval. The function $\Gamma_{\sigma}: \mathbb{R} \mapsto \mathbb{R}$ denotes the gaussian kernel weighting the influence of data points. Parameter $\hat{s}_{i}$ refers to the projection of data point $\mathbf{p}_{i}$ onto the centerline curve as shown below. The fixed start and end points of the curve are $\mathbf{x}_{0}$ and $\mathbf{x}_{1}$. The cost functional is regularized by the second and third term, weighted with factors $\lambda$ and $\mu$, respectively.

$$
\begin{aligned}
I(\mathbf{c}, r)= & \int_{0}^{1} \frac{1}{N} \sum_{i=1}^{N} \Gamma_{\sigma}\left(s-\hat{s}_{i}\right)\left(\left\|\mathbf{c}(s)-\mathbf{p}_{i}\right\|-r(s)\right)^{2} \mathrm{~d} s+ \\
& \lambda \int_{0}^{1} \frac{1}{2}\|\nabla \mathbf{c}(s)\|^{2} \mathrm{~d} s+\mu \int_{0}^{1} \frac{1}{2} r^{\prime}(s)^{2} \mathrm{~d} s \\
\mathbf{c}(0)= & \mathbf{x}_{0}, \quad \mathbf{c}(1)=\mathbf{x}_{1}, \quad \hat{s}_{i}=\arg \min _{s}\left\|\mathbf{c}(s)-\mathbf{p}_{i}\right\|
\end{aligned}
$$

The corresponding Euler-Lagrange equations give rise to the gradient descent equations

$$
\begin{gathered}
\frac{\partial \mathbf{c}}{\partial t}(s)=-\frac{1}{N} \sum_{i=1}^{N} \Gamma_{\sigma}\left(s-\hat{s}_{i}\right)\left(\left\|\mathbf{c}(s)-\mathbf{p}_{i}\right\|-r(s)\right) \frac{\mathbf{c}(s)-p_{i}}{\left\|\mathbf{c}(s)-p_{i}\right\|}-\lambda \Delta_{s} \mathbf{c}(s) \\
\text { and } \frac{\partial r}{\partial t}(s)=-\frac{1}{N} \sum_{i=1}^{N} \Gamma_{\sigma}\left(s-\hat{s}_{i}\right)\left(\left\|\mathbf{c}(s)-\mathbf{p}_{i}\right\|-r(s)\right)-\mu \frac{\partial^{2}}{\partial s^{2}} r(s) .
\end{gathered}
$$

Defining a reference frame. When sweeping a $2 \mathrm{D}$ shape along a $3 \mathrm{D}$ space curve, one has to define the frame of reference, i.e. the local coordinate system for the cross-section function. This is important for building a 3D mesh and for generalizing the cross-section function.

A geometrically straightforward choice is the Frenet-Serret reference frame, as it was used in [15. It defines the local coordinate system by the tangent, normal, and binormal vectors of the spine curve. Let again $\mathbf{c}$ denote the spine curve; we assume natural parametrization for simplicity. Then the basis vectors of the Frenet-Serret frame are defined as

$$
\mathbf{Z}=\frac{\mathrm{d} \mathbf{c}}{\mathrm{d} s}, \quad \mathbf{X}=\frac{\frac{\mathrm{d} \mathbf{Z}}{\mathrm{d} s}}{\left\|\frac{\mathrm{d} \mathbf{Z}}{\mathrm{d} s}\right\|}, \quad \text { and } \quad \mathbf{Y}=\mathbf{Z} \times \mathbf{X} .
$$


As mentioned above, this formulation suffers from instabilities of the second derivative, which may lead to discontinuities. We employ here a less general, but more stable reference frame. We choose a fixed vector $\mathbf{W}$ that is known to be non-collinear to the spine curve at any point. This can be done by applying prior knowledge of the organ to be modeled; in our case we chose $\mathbf{W}$ to be the normal vector of the coronal plane, in which the aorta roughly stretches. The basis vectors are then defined by

$$
\mathbf{Z}=\frac{\mathrm{d} \mathbf{c}}{\mathrm{d} s}, \quad \mathbf{X}=\mathbf{Z} \times \mathbf{W}, \quad \text { and } \quad \mathbf{Y}=\mathbf{Z} \times \mathbf{X}
$$

\section{Results}

Segmentation and model fitting was tested on real time TrueFISP (SSFP) MR image series acquired orthogonal to the long axis of the aorta in three healthy volunteers, with TE 1.48, TR 2.96, FL 70, and acquisition matrix size ranging from $88 \times 128$ to $138 \times 192$; pixel size was $1.77 \times 1.77 \mathrm{~mm}$, slice thickness 6 $\mathrm{mm}$. The scans were performed on a Siemens $1.5 \mathrm{~T}$ Espree with parallel imaging (GRAPPA factor 2).

\subsection{Segmentation Accuracy}

The $2 \mathrm{D}$ segmentation was performed on a total of 2416 slices, some exhibiting low resolution, severe noise, or blood flow artifacts. Evaluation by a clinical expert showed no or very minor deviations from the true contour in $98.3 \%$ of the cases, while the remaining $1.7 \%$ were still rated clinically usable. Segmentation time per slice was less than 1 msec.

\subsection{Model Fitting with Synthetic Data}

To assess the accuracy of the model optimization procedure we first used a synthetically generated point cloud of known properties as data base. Uniformly distributed points were sampled on the surface of an idealized aorta model. The centerline was discretized in 35 piecewise linear segments and initialized as a straight line between start and end point. The radius function was initialized zero everywhere. Some sample images of the model after various numbers of iterations are shown in figure 3 .

Model fitting was carried out for different numbers of data points, the results are shown in the table below. The detected centerline was very close to the theoretical position with a mean square error (MSE) around $10^{-5}$ (spine length $\approx 2.57$ ). Denser point clouds led to a more precise result.

\begin{tabular}{c|cccc} 
number of data points & 100 & 200 & 500 & 1000 \\
\hline MSE $\times 10^{-5}$ & 4.622 & 3.148 & 2.770 & 2.576
\end{tabular}




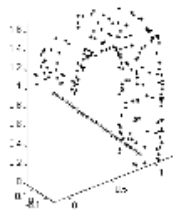

0 iterations

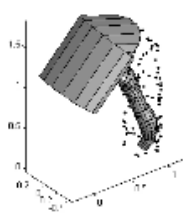

1 iteration

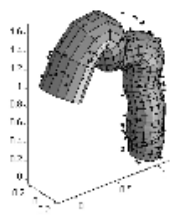

5 iterations

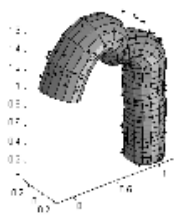

10 iterations
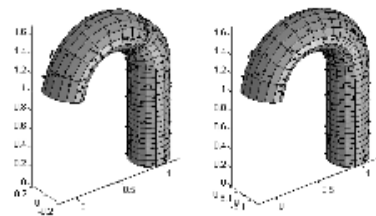

20 iterations

30 iterations

Fig. 3. Model fitting progress for synthetic data set of 200 points

\subsection{Model Fitting with Real Data}

Finally we evaluated the accuracy of the model on real data. Consecutive image slices were cut out from a 4D data volume and the aortic cross-section was segmented using the method described in section 2.1. The resulting model and a sample endoscopic view of it are shown in figure 4. Visual inspection showed good correspondence in most areas. Outliers did not have a big influence on the result, which is due to the smooth structure of the aorta and the model.
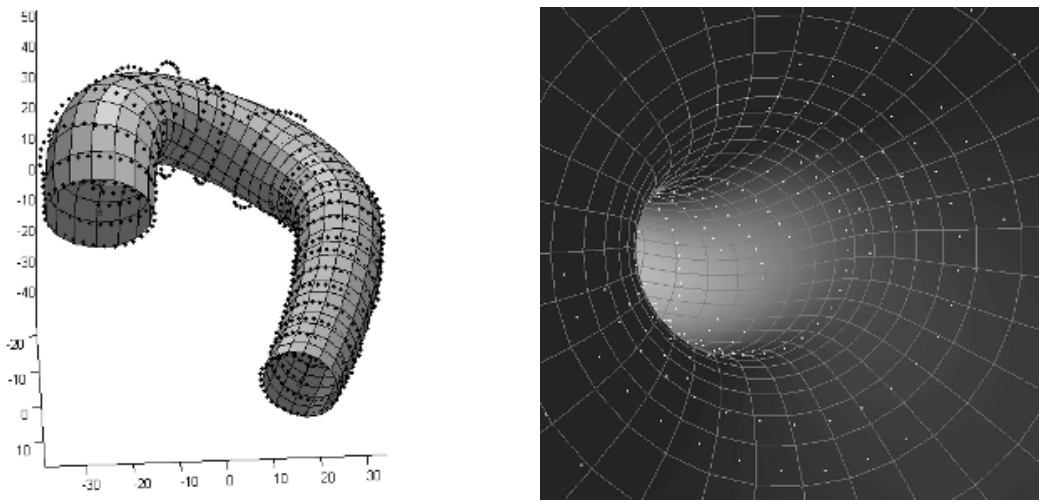

Fig. 4. Aorta model created from real human MRI data (left) and sample virtual endoscopic view (right)

\section{Discussion and Future Work}

We presented a method for segmenting and modeling the human aorta suitable for real-time virtual endoscopy. The highly optimized Geodesic Active Contour segmentation allows processing of an image slice in less than $1 \mathrm{msec}$. We derived and tested a variational fitting scheme for a generalized cylinder model to a dynamic point cloud. Furthermore, a new local reference frame was implemented for the model. The segmentation and model can now be integrated into the real time acquisition and feedback system described in [5].

We plan to extend the GC model by attaching a finite element mesh to the tubular GC surface to better model local deformations, which will be especially 
important when applying the method to structures with more complex shape such as the left atrium.

The influence of motion was neglected in our model so far. Possible sources of displacements are the cardiac and respiratory cycles, as well as patient movement during the intervention. To cope with the periodic cardiac motion, we are extending the model to 4D with imposed constraints on timepoint-to-timepoint displacement. When addressing respiratory and external patient motion, one must consider in-plane as well as through-plane displacements. Respiratory gating methods during the acquisition as well as registration and/or quality control measures may be required depending on the exact application. And finally, we plan to develop an update strategy for the real time model based on local geometry and phase of the interventional procedure.

\section{References}

1. Zhang, Q., Wendt, M., Aschoff, A., Zheng, L., Lewin, J., Duerk, J.: Active MR guidance of interventional devices with target-navigation. Magn Reson Med 44(1) (2000) 56-65

2. Nayak, K.S., Pauly, J.M., Nishimura, D.G., Hu, B.S.: Rapid ventricular assessment using real-time interactive multislice MRI. Magn Reson Med 45(3) (2001) 371-375

3. Serfaty, J.M., Atalar, E., Declerck, J., Karmakar, P., Quick, H.H., Shunk, K.A., Heldman, A.W., Yang, X.: Real-time projection MR angiography: feasibility study. Radiology 217(1) (2000) 290-295

4. Guttman, M., Lederman, R., Sorger, J., McVeigh, E.: Real-time volume rendered MRI for interventional guidance. J Cardiovasc Magn Reson 4(4) (2002) 431-42

5. Kirchberg, K.J., Wimmer, A., Lorenz, C.H.: Real-Time Virtual Endoscopy for MR-Guided Aortic Interventions. In: Proc. 14th ISMRM. (2006) 268

6. Binford, T.: Visual perception by computer. In: IEEE Conference on Systems and Control. (1971)

7. Caselles, V., Kimmel, R., Sapiro, G.: Geodesic active contours. International Journal of Computer Vision 22(1) (1997) 61-79

8. Perona, P., Malik, J.: Scale-Space and Edge Detection Using Anisotropic Diffusion. IEEE Trans. Pattern Anal. Mach. Intell. 12(7) (1990) 629-639

9. Weickert, J., Kuehne, G.: Fast methods for implicit active contour models. In Osher, S., Paragios, N., eds.: Geometric Level Set Methods in Imaging, Vision, and Graphics. Springer (2003) 43-57

10. Weickert, J., ter Haar Romeny, B., Viergever, M.: Efficient and reliable schemes for nonlinear diffusion filtering. IEEE Trans. Imag. Proc. 7(3) (1998) 398-410

11. Adalsteinsson, D., Sethian, J.: A fast level set method for propagating interfaces. Journal of Computational Physics 118(2) (1995) 269-277

12. Goldenberg, R., Kimmel, R., Rivlin, E., Rudzsky, M.: Fast geodesic active contours. IEEE Transactions on Image Processing 10(10) (2001) 1467-1475

13. Krissian, K., Westin, C.: Fast and accurate redistancing for level set methods. Computer Aided Systems Theory (EUROCAST'03) (2003) 48-51

14. McInerney, T., Terzopoulos, D.: Deformable models in medical image analysis: a survey. Med Image Anal 1(2) (1996) 91-108

15. O'Donnell, T., Boult, T., Fang, X.S., Gupta, A.: The extruded generalized cylinder: a deformable model for object recovery. In: CVPR. (1994) 174-181

16. O'Donnell, T., Gupta, A., Boult, T.E.: A new model for the recovery of cylindrical structures from medical image data. In: CVRMed-MRCAS '97. (1997) 223-232 